\title{
RANK/RANKL EXPRESSION IS INDUCED BY CARDIAC SURGICAL OPERATION
}

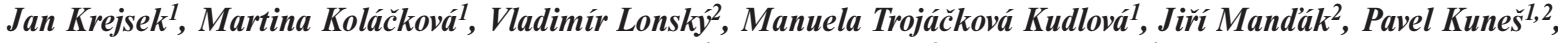 \\ Karolina Jankovičová ${ }^{1}$, Dana Vlášková ${ }^{2}$, Ctirad Andrýs ${ }^{1}$
}

Charles University in Prague, Faculty of Medicine and University Hospital Hradec Králové, Czech Republic: Department of Clinical Immunology and Allergology ${ }^{1}$, Department of Cardiac Surgery ${ }^{2}$

\begin{abstract}
Summary: Background: Cardiac surgery provokes a systemic inflammatory response in any patient. This complex body reaction involves also RANK/RANKL molecules which have been recently identified as principal regulators of bone metabolism. Aims: To follow the changes in the expression of RANK/RANKL molecules on innate immune cells of cardiac surgical patients. Patients and Methods: Twenty-six patients undergoing cardiac surgical were assigned to undergo coronary artery bypass grafting using either cardiopulmonary bypass ("on-pump") or modified "miniinvasive on-pump". The expression of RANK/RANKL was performed by flow cytometry. Results: Significantly increased expression of RANK on monocytes of "miniinvasive on-pump" patients was found at the $1^{\text {st }}$, the $3^{\text {nd }}$, and $7^{\text {th }}$ postoperative days. The similar pattern was found also for monocyte RANKL expression. In addition, RANKL expression was significantly increased at the $3^{\text {rd }}$ postoperative day in "on-pump" patient. No significant differences between "miniinvasive on-pump" and "on-pump" cardiac surgical patients were found. Conclusion: The expression of both RANK and RANKL molecules is significantly enhanced on monocytes of "miniinvasive on-pump" cardiac surgical patients.
\end{abstract}

Key words: RANK; RANKL; Monocytes; Cardiac surgery; Cardiopulmonary bypass

\section{Introduction}

Cardiac surgical operation provokes a systemic inflammatory response in any patient. This inflammatory response is a result of very complex interplays based on both inherited individual predispositions and many variables including extent of body trauma, impact of cardiopulmonary bypass, and ischemia-reperfusion injury to list the most important (4). Such adverse conditions are accompanied by the development of danger patterns which are identified by a limited number of so called pattern recognition receptors (PRR). Both immune cells and cells of non immune origin are activated via PRR receptor with subsequent release of various proinflammatory mediators, including cytokines with proinflammatory activities, such as TNF $\alpha$, IL-1, and chemokines. Their contribution to the development and maintainance of systemic inflammatory response (SIRS) in cardiac surgical patients is well established now $(2,14,16)$. The modulation of both innate $(9,11)$ and specific immune response (7) were followed in cardiac surgical patients also by our group.

The impact of cardiac surgery is really pluripotent affecting numerous body systems. However, although the participation of inflammatory mediators in the cardiac surgery has become widely recognized, identification and characte- rization of other actors of inflammation is still awaited. Receptor activator of nuclear factor-KB ligand (RANKL) and its receptor RANK are among such promising molecules. These molecules are members of TNF receptor superfamily. They are either expressed as membrane receptors or are shed in a soluble form into the body fluids. These factors have previously been indentified as essential mediators for paracrine signalling in bone metabolism $(12,17)$. However, their contribution to the body homeostasis is much complex. It has recently been confirmed that RANK and RANKL are also involved in modulation of the immune response through interaction with both innate and adaptive immunity cells, such as dendritic cells, macrophages, $T$ and B cells, respectively $(1,3)$.

Furthermore, there is an increasing number of reports regarding RANK/RANKL involvement in some cardiovascular disorders such as acute coronary syndrome (18), acute myocardial infarction and heart failure (6). However, there is an apparent lack of informations to which extent is RANK/RANKL signalling system modulated in patients undergoing cardiac surgery. We found in this our pilot study that there was a significant increase in the expression of both RANK and RANKL on monocytes of cardiac surgical patients operated using "miniinvasive“ cardiopulmonary bypass during an early postoperative period. 


\section{Patients}

Twenty-six patients ( 4 females and 22 males) were enrolled to this study. They were assigned by the cardiac surgeon outside of research team to undergo coronary artery bypass grafting $(\mathrm{CABG})$ using either cardiopulmonary bypass (CPB), „on-pump“ surgery or miniinvasive $\mathrm{CPB}$, „miniinvasive on-pump“ surgery. Thirteen patients underwent „on-pump“ surgery ( 3 females, 10 males; mean age 66.2 \pm 8.3 years). Thirteen patients underwent „miniinvasive on-pump“ surgery ( 1 female, 12 males; mean age $65.8 \pm 8.6$ years).

Patients in both groups were comparable in age and preoperative ejection fraction. In „on-pump“ patients ejection fraction was 61 , range 53.5 to 68.3 ; median of anastomoses was 2.5. In „miniinvasive on-pump“ patients ejection fraction was $60,49.5$ to 70 ; median of anastomoses was 2 . No significant differences were found between groups regarding duration of CPB.

The exclusion criteria were concomitant surgery (valvar or aortic), an emergency procedure, patients with local or systemic infection or inflammation, severe left ventricular dysfunction (ejection fraction $<30 \%$ ), renal failure (serum creatinine $>180 \mu \mathrm{mol} . \mathrm{l}^{-1}$ or active renal replacement therapy). The potential enrollee needed to meet the criteria for „on-pump“, and „miniinvasive“ CPB procedures.

Elective patients discontinued antiplatelet agents, aspirin $100 \mathrm{mg}$ in one daily dose, at least five days prior to surgery. Each subject passed a sreening examination including medical history, physical examination, blood and urine test, ECG, X-ray of the chest and echocardiography.

\section{Anaesthetic management}

Food and fluid intake was discontinued at midnight on the day preceding surgery.

Anaesthesia was induced with intravenous thiopenthal or midazolam and sufentanyl, muscle relaxation with cisatracurium and was maintaned by infusion of cisatracurium, sufentanyl and propofol. Isoflurane was added in oxygen.

All patients were monitored according to general protocol used worldwide during open heart procedures. Median sternotomy was a routine surgical approach in all cases. The left internal mammary artery and great saphenous vein were harvested.

\section{„On-pump“ surgery}

After median sternotomy and pericardotomy cardiopulmonary bypass was established by standard aortic cannulation and two-stage venous cannulation of the right atrium. Target ACT time was over 480 seconds. Cardiac arrest was instituted by antegrade infusion of cold crystalloid cardioplegia (St. Thomas solution, Ardeapharma, Sevetin, Czech Republic) or cold blood cardioplegia (blood to St. Thomas solution in ratio $4: 1$ ), repeated every 20 minutes, and topical cooling for myocardial protection were employed.
The extracorporeal circuit consisted of membrane oxygenator (Polystan Safe Maxi, Maquet Cardiopulmonary AG, Hirrlingen, Germany) and roller pump with non-pulsatile flow (Stőckert S3, Sorin Group, München, Germany). Oxygenator and tubing were primed with a mixture of Hartmann's solution, 10\% Rheodextran solution (molecular weight 40,000), 10\% Mannitol solution, $8.4 \%$ Sodium bicarbonate, Magnesium sulphure, 5,000 IU of heparin. Normothermic perfusion with calculated blood flow 2.4 $\mathrm{ml} . \mathrm{l}^{-1} \cdot \mathrm{m}^{-2}$ was used.

Once completing all distal anastomoses, the aortic cross-clamp was removed and the proximal anastomosis were performed with tangential aortic clamp.

\section{„Miniinvasive on-pump“ surgery}

„Miniinvasive on-pump“ surgery was established using a small $22 \mathrm{~F}$ two-stage venous drainage and ascending aortic return. Minisystem Synergy Sorin ${ }^{\circledR}$ (Dideco S.p.A., Mirandola, Italy) was used.

Oxygenator and tubing were primed with a mixture of $500 \mathrm{ml}$ Ringer's lactate, $5000 \mathrm{IU}$ heparin, $80 \mathrm{ml}$ natrium bicarbonate $\left(\mathrm{NaHCO}_{3} 8,4 \%\right), 20 \mathrm{ml}$ magnesium sulphate $10 \%$, manitol $1 \mathrm{~g} / \mathrm{kg}$ body weight.

Normothermic perfusion with target ACT above $480 \mathrm{~s}$ and Calafiore cardioplegic arrest was used. All patients received an internal artery mammary graft to the left anterior descending coronary artery (LAD). The central aorto-venous anastomoses were performed during the reperfusion phase of CPB with the heart beating.

\section{Ethics Commitee approval}

The study protocol was approved by the Ethics Committee of the University Hospital in Hradec Králové. All participants were informed in detail about the purpose of the study both orally and in writing. They were free to ask any questions. All active subjects have given written informed consent.

\section{Blood sampling}

Peripheral venous blood from an antebrachial vein was withdrawn in the operating room and in the intensive care unit. Samples were collected into tubes Vacutainter treated with lithium heparin, manufactured by Becton Dickinson, UK.

In all „on-pump“, and „miniinvasive on-pump“ groups of patients, blood was withdrawn at following time points:

1) introduction to anaesthesia, which in both groups represented the baseline or reference value for all parameters measured thereafter

2) the termination of operation

3) the first postoperative day

4) the third postoperative day

5) the seventh postoperative day 


\section{Methods}

Double immunofluorescence standard whole blood staining method was used. Briefly, $25 \mu \mathrm{l}$ of heparinized venous was incubated with given pair of monoclonal antibodies $(2 \times 3 \mu 1)$ for 20 minutes at room temperature. After subsequent lysis (10 min) of red blood cell (Optilyse C, Immunotech, France) samples were washed by buffered saline solution (PBS) and resuspended in PBS with azide. Following combination of monoclonal antibodies labeled either with fluoresceine isothiocyanate (FIC) or phycoerythrine (PE) were used: CD45-FITC/CD14-PE, isotypic control IgG1-fITC/IgG2a-PE (Immunotech, France). Monoclonal antibodies reacting with RANK (CD265, Receptor Activator of $\mathrm{NF \kappa B}$ ) was $\operatorname{IgG} 2$ a rat monoclonal antibody, clone R12-31 PE purchased from eBioscience, USA. Monoclonal antibodies reacting with RANKL (CD265, Receptor Activator of NFKB Ligand) was IgG2b mouse monoclonal antibody, clone MIH24 PE purchased from eBioscience, USA. Measurements were performed using FACSCalibur flow cytometer and data acquired by CellQuest software (BD Bioscience, NY, USA). Lymphocytes, monocytes and granulocytes were identified on the basis of different CD45 v. CD14 expression (leukogate). Results of flow cytometric analysis were expressed as a median fluorescence intensity (MFI) for a given population.

\section{Statistical analysis}

Within group differences were evaluated by comparison of RANK/RANKL expression separately for monocytes and granulocytes expressed as MFI using Friedman ANOVA and Wilcoxon pair test. Differences between the groups of patients were tested using Kruskal-Wallis ANOVA. Bonferroni correction was applied when Wilcoxon test and Kruskal-Wallis ANOVA were used for multiple comparisons. Clinical data were analyzed by Fisher exact test, Mann-Whitney U test and t-test.

Differences were considered significant at $\mathrm{p}<0.05$.

Data are expressed as medians and interquartile ranges in plots. Plots also display the range of non-outliers values.

\section{Results}

Dynamics in the expression of RANK (CD265) separately for monocytes and granulocytes were followed in „onpump“ and „mini on-pump“ cardiac surgical patients after surgery and during an early postoperative period. Results are expressed as percentage of changes of RANK MFI and compared to the preoperative value which is considered as baseline. The expression of RANK on both monocytes and granulocytes was decreased after operation being significant in former one followed by significant increase during an early postoperative period in „mini on-pump“ patients only, being without any change in „on-pump“ patients. These changes were more prominent in monocytes compared to granulocytes. There was no significant differences between „on-pump“ and „mini on-pump“ patients. Results are show in Fig. 1, 2.

Dynamics in the expression of RANKL (CD254) are shown in Figs. 3 and 4. There were no significant changes in the expression of RANKL on granulocytes of cardiac surgical patients. In contrast, monocyte RANKL expression was significantly increased after surgery and during postoperative period in „mini on-pump“ patients, being increased at the $3^{\text {rd }}$ postoperative day in „on-pump“ patients as well. There were no significant differences between patients groups suggesting that the very surgical trauma is responsible for the induction of RANK/RANKL on leukocytes of cardiac surgical patients being minimally influenced by CPB.

\section{Discussion}

Current view of an inflammatory response induced by cardiac surgery is more and more complex. New markers are followed not only to understand better to the pathophysiology of this reaction but their putative clinical value is also tested. RANK/RANKL molecules and the decoy receptor osteoprotegrin represent a novel triad with pleiotropic effects on bone metabolism, immune system, and inflammatory response $(12,17)$. Recently, it has been found that RANK/RANKL regulatory axis have an important role in the immunopathogenesis of vascular diseases.

Sandberg et al (18) reported increased expression of RANKL on monocytes of unstable angina patients. Furthermore, RANKL enhanced the release of monocyte chemoattractant protein in mononuclear cells from unstable angina, and promoted matrix metaloproteinase activity. Persistent inflammation appears to play a role in the development of heart failure. In addition to TNF $\alpha$, several other members of TNF $\alpha$ or TNF $\alpha \mathrm{R}$ superfamilies such as RANK/RANKL also could be involved in the development of myocardial failure. Indeed, Ueland et al (20) found increased systemic expression of both RANKL on T cells and sRANKL in serum of patients with myocardial failure.

To underline a broad physiological role of RANK, its expression is ubiquitous comprising skeletal muscle, liver, gut, thymus, adrenal gland. It is also expressed on a majority of immune cells, such as dendritic cells, monocytes, B cells, NK cells, and granulocytes (3). RANKL is expressed on dendritic cells, T cells, monocyte/macrophage cells. Expression of both RANK and RANKL is inducible in the presence of proinflammatory cytokines, such as TNF $\alpha$, IL$1 \beta$, GM-CSF, and chemokines. RANKL expression is further induced by glucocorticoids (19).

Numerous proinflammatory stimuli are raised by cardiac surgery as a result of local trauma, cardiopulmonary bypass as well as pulmonary and myocardial reperfusion (4). In an attempt to reduce the inflammatory response which is inseparable linked to any cardiac surgery, procedures either avoiding cardiopulmonary bypass or mini- 


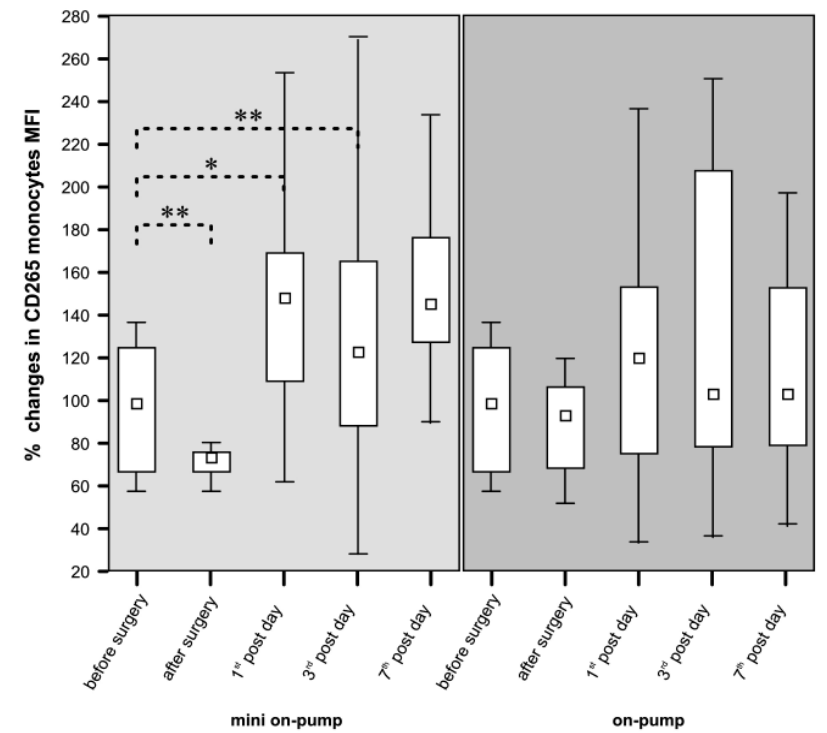

Fig. 1: Comparison of \% changes in CD265 monocyte MFI between mini on-pump patients and on-pump patients.

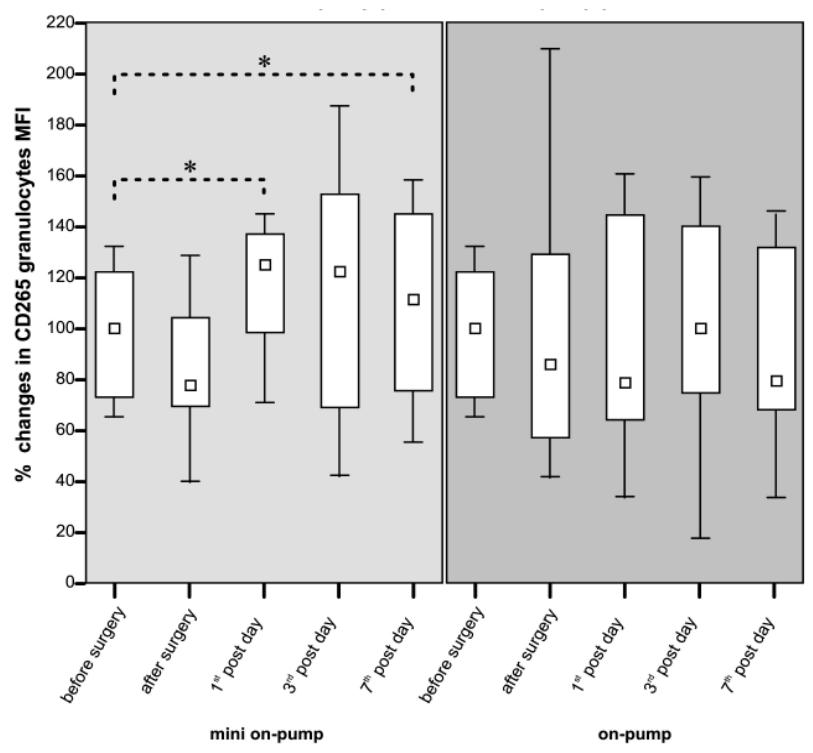

Fig. 2: Comparison of \% changes in CD265 granulocyte MFI between mini on-pump patients and on-pump patients.

mizing its adverse effects are now extensively studied (5). Our study was addressed two aims. The first one was to follow the changes in the expression of RANK and RANKL on monocytes and granulocytes of patients undergoing cardiac surgery. The second aim was to test wheather there are differences in the RANK/RANKL expression in patients undergoing $\mathrm{CABG}$ using standard $\mathrm{CPB}$ or modified „miniinvasive" CPB. Suprisingly, there was more significant dynamics in the expression of both RANK, RANKL in „miniinvasive“ CPB patients compared to „standard“ $\mathrm{CPB}$ patients in whom only nonsignificant changes were found.

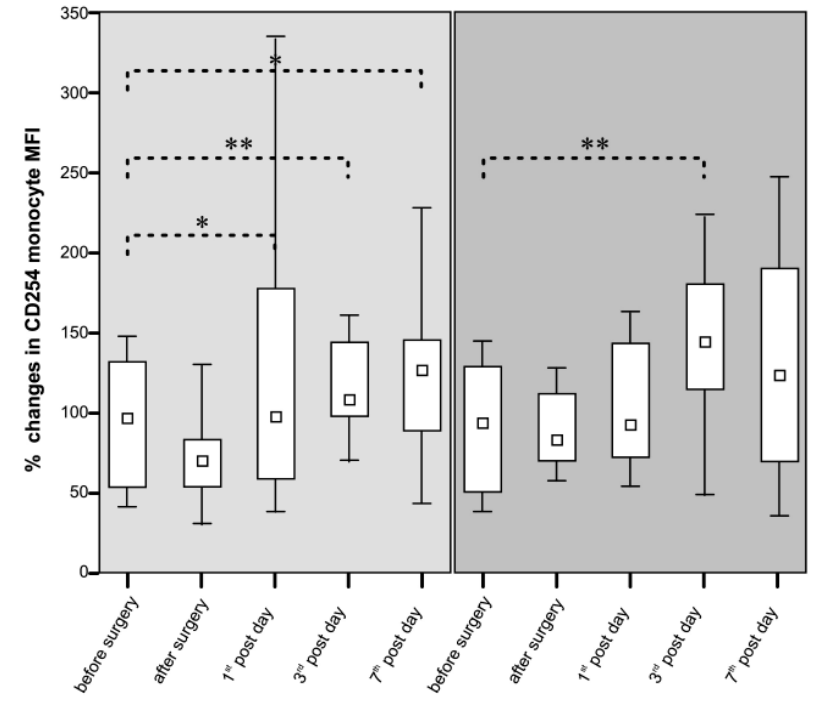

mini on-pump

on-pump

Fig. 3: Comparison of \% changes in CD254 monocyte MFI between mini on-pump patients and on-pump patients.

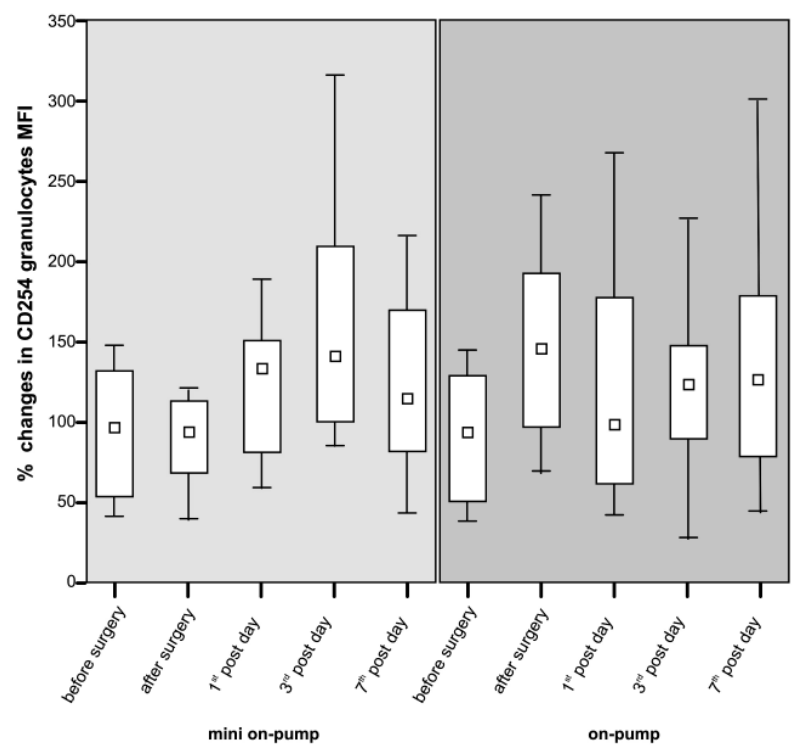

Fig. 4: Comparison of \% changes in CD254 granulocyte MFI between mini on-pump patients and on-pump patients.

Cells of monocyte/macrophage origin are considered as sentinel cells sensing danger signals by their surveillance receptors, such as TLR receptors, in the onset of inflammatory reaction. In opposite, granulocytes are the principal effector inflammatory cells. Based on this paradigm the expression of both RANK and RANKL is significantly increased on monocytes of cardiac surgical patients after surgery and during postoperative period. This phenomena is linked to overall monocytes activation as other surface molecules with both proinflammatory potential such as TLR receptors (10) and antiinflammatory capacity such as sca- 
venger receptor for hemoglobin display the similar pattern (8).

As data regarding changes in the expression of RANK/ RANKL during surgery and in an early postoperative period are entirely absent it is difficult to disscus them in the context of other works. Increased RANK/RANKL expression could contribute to inflammation, leukocyte recruitment and matrix degradation as described in acute coronary syndrome by Sandberg et al (18). This was clearly proved by an excellent clinical and experimental study by Poubelle et al. (15) focused on neutrophils RANK/RANKL expression in rheumatoid arthritis. They found that neutrophils influenced by proinflammatory substances are expressing both RANK/RANKL. RANK/RANKL expression is induced by TNFo, GM-CSF, and IL-4 in vitro.

It might be hard to draw a firm conclusion from this pilot study. It is apparent from our results that RANK/ RANKL expression, especially on monocytes, is modulated by cardiac surgical operation. Wheather it is the regulatory attempt to modulate inflammatory response as shown e.g. in animal model of CD40L - deficient mice (13) or it is the only epiphenomena remains to be established.

\section{Acknowledgment}

This work was supported by Ministry of Education, Czech Republic, project No. MSM0021620812 and Internal Grant Agency of Ministry of Health, Czech Republic, No. NR/9090-4.

\section{References}

1. Anandarajah AP, Schwarz EM. Anti-RANKL therapy for inflammatory bone disorders: mechanisms and potential clinical applications. J Cell Biochem 2006;97:226-32.

2. Anselmi A, Abbate A, Girola F, et al. Myocardial ischemia, stunning, inflammation, and apoptosis during cardiac surgery: a review of evidence. Eur J CardioThor Surgery 2004;25:304-11.

3. Atkins GJ, Kostakis P, Vincent C, et al. RANK expression as a cell surface marke of human osteoclast precursors in peripheral blood, bone marrow, and giant cell tumors of bone. J Bone Miner Res 2006;21:1339-49.
4. Franke A, Lante W, Fackeldey V, et al. Pro-inflammatory cytokines after different kinds of cardio-thoracic surgical procedures: is what we see what we know? Eur J Cardio-Thor Surgery 2005;28:569-75.

5. Chowdhury UK, Malik V, Yadav R, et al. Myocardial injury in coronary artery bypass grafting: On-pump versus off-pump comparison by measuring high-sensitivity C-reactive protein, cardiac troponin I, heart-type fatty acid-binding protein, creatine kinase-MB, and myoglobin release. J Thorac Cardiovasc Surg 2008; 135:1110-9.

6. Crisafulli A, Micari A, Altavilla D, et al. Serum levels of osteoprotegerin and RANKL in patients with ST elevation acute myocardial infarction. Clin Science 2005;109:389-95.

7. Jankovičová $\mathrm{K}$, Trojáčková Kudlová $\mathrm{M}$, Koláčková $\mathrm{M}$, et al. The effect of cardiac surgery on peripheral blood lymphocyte populations. Acta Med 2008;51:25-9.

8. Koláčková M, Kudlová M, Kuneš P, et al. Early expression of FçRI (CD64) on monocytes of cardiac surgical patients and higher density of monocyte anti-inflammatory scavenger CD163 receptor in „on-pump“ patients. Med Inflamm 2008;235461.

9. Koláčková M, Trojáčková Kudlová $\mathrm{M}$, Lonský $\mathrm{V}$, et al. The expression of CD38 ADP-ribosyl cyclase ectoenzyme in immune cells of cardiac surgical patients. Acta Med 2008;51:31-5.

10. Krejsek J, Kuneš P, Koláčková M, et al. The expression of Toll-like receptor 2 and 4 on innate immunity cells is modulated by cardiac surgical operation. Scand J Clin Lab Invest 2008;68:749-58.

11. Kudlová M, Koláčkova M, Kuneš $\mathrm{P}$, et al. Expression of an activated form of in tegrin $\beta_{2}$ chain CD18 in cardiac surgical operations. Acta Med 2007;50:187-93.

12. Lorenzo J, Horowitz M, Choi Y. Osteoimmunology: interactions of the bone and immune system. Endocrine Reviews 2008;29:403-40.

13. Padigel UM, Kim N, Choi Y, Farrell JP. TRANCE-RANK costimulation is required for IL-12 production and the initiation of a Th1-type response to Leishmania major infection in CD40L-deficient mice. J Immunol 2003;171: 5437-41.

14. Paparella D, Yau TM, Young E. Cardiopulmonary bypass induced inflammation: pathophysiology and treatment. An update. Eur J Cardio-Thor Surgery 2002;21:232-44

15. Poubelle PE, Chakravarti A, Fernandes MJ, Doiron K, Marceau AA. Differential expression of RANK, RANKL, and osteoprotegerin by synovial fluid neutrophils from patients with rheumatoid arthritis and by healthy human blood neutrophils. Arthritis Research Therapy 2007;9:R25.

16. Rastan AJ, Bittner HB, Gummert JF, et al. On-pump beating heart versus offpump coronary artery bypass surgery-evidence of pump-induced myocardial injury. Eur J Cardio-Thor Surgery 2005;27:1057-64.

17. Rauner M, Sipos W, Pietschmann P. Osteoimmunology. Int Arch Allergy Immunol 2007;143:31-48.

18. Sandberg WJ, Yndestad A, Oie E, et al. Enhanced T-cell expression of RANK ligand in acute coronary syndrome. Arterioscler Thromb Vasc Biol 2006;26: 857-63.

19. Ueland T, Gullestad L, Simonsen S, et al. Decreased endomyocardial RANKL expression in transplant coronary artery disease. Transplantation 2006;81: $1467-1470$.

20. Ueland T, Yndestad A, Oie E, et al. Dysregulated osteoprotegerin/RANK ligand/RANK axis in clinical and experimental heart failure. Circulation $2005 ; 111: 2461-8$

Received: 03/07/2009.

Accepted in revised form: 06/10/2009.

Corresponding author:

Prof. RNDr. Jan Krejsek, CSc., Department of Clinical Immunology and Allergology, University Hospital, Sokolská 581, 50005 Hradec Králové, Czech Republic; e-mail: krejsek@fnhk.cz 